\title{
EL NACIMIENTO DE \\ LA FACULTAD DE \\ ECONOMÍA DE LA \\ UNIVERSIDAD DE \\ NUEVO LEÓN (1957) \\ Y LA INTEGRACIÓN \\ DEL CURSO DE \\ CIVILIZACIÓN \\ CONTEMPORÁNEA \\ DE LA UNIVERSIDAD \\ DE COLUMBIA \\ José Rafael González Díaz*
}

RESUMEN: La Universidad de Columbia determinó el 20 de enero de 1919 implantar el curso Contemporary Civilization, que se convirtió con el paso del tiempo en uno de los más exitosos de la educación estadounidense. El propósito del curso era comprender los problemas del mundo y sus soluciones. Solo un estudiante cultivado puede alcanzar un entendimiento profundo de sí mismo y de la sociedad, y de emitir sus propios juicios. El proyecto se inspiró en la idea de la obligación moral de ser inteligente de John Erskine. Daniel Cosío Villegas (1898-1976) impulsó la introducción del curso en México en el nuevo plan de estudios (1958) de la Facultad de Economía de Nuevo León. En esta investigación exploramos las circunstancias históricas del curso y los problemas que enfrentó Consuelo Meyer L'Epée (1918-2010) para implantarlo.

\author{
rose \\ THE BIRTH OF THE SCHOOL OF ECONOMICS AT THE UNIVERSITY \\ OF NUEVO LEÓN (1957) AND THE INTEGRATION OF THE CONTEMPORARY \\ CIVILIZATION COURSE AT COLUMBIA UNIVERSITY
}

ABSTRACT: On January 20, 1919, Columbia University decided to introduce the Contemporary Civilization course. This new academic program became over time one of the most successful in the American education. The purpose of the course was to understand the world's problems and their solutions. Only a cultivated student can develop a deep understanding of himself and society, and the ability to make his or her own judgments. The project was inspired by John *Departamento Académico de Estudios Generales, ITAM. 
Erskine's (1879-1951) the moral obligation to be intelligent. Daniel Cosío Villegas (1898-1976) promoted the introduction of the Contemporary Civilization course in Mexico in the new curriculum (1958) of the Faculty of Economics of Nuevo León. In this research we explore the historical circumstances of this course and the challenges faced by Consuelo Meyer L'Epée (1918-2010) in its implementation.

PALABRAS ClaVE: Consuelo Meyer L'Epée, Daniel Cosío Villegas, enseñanza de la economía en México, historia de la educación en México.

KEY WORDs: Consuelo Meyer L'Epée, Daniel Cosío Villegas, economics teaching in Mexico, history of education in Mexico.

RECEPCIÓN: 4 de febrero de 2021.

APROBACIÓN: 23 de marzo de 2021.

DOI: $10.5347 / 01856383.0137 .000299735$ 


\section{EL NACIMIENTO DE \\ LA FACULTAD DE ECONOMÍA DE LA \\ UNIVERSIDAD DE \\ NUEVO LEÓN (1957) \\ Y LA INTEGRACIÓN \\ DEL CURSO DE \\ CIVILIZACIÓN \\ CONTEMPORÁNEA \\ DE LA UNIVERSIDAD \\ DE COLUMBIA*}

\section{Introducción}

El estudio formal y sistemático de la economía en México no fue posible hasta que se instauró la Sección de Economía en la Facultad de Derecho y Ciencias Sociales de la Universidad Nacional en 1929. ${ }^{1}$ Hasta ese momento casi todos los "economistas" eran ingenieros, agrónomos, contadores o abogados que habían adquirido conocimientos económicos en la práctica profesional o de manera autodidacta, y solo un reducido grupo había realizado estudios

*Este artículo se basó en la ponencia presentada en el VII Simposio de la Red Internacional de Estudios Generales celebrada en el Instituto Tecnológico de Santo Domingo en la República Dominicana, que se llevó a cabo del 4 al 6 de noviembre de 2015. Dedico este trabajo a Sofía y Josué Márquez González, jóvenes que hacen realidad la obligación moral de ser inteligentes.

${ }^{1}$ Francisco Rodríguez Garza y Santiago Ávila Sandoval, "La enseñanza y la difusión de la economía en el periodo de entreguerras”, Análisis económico XV, núm. 31 (2000): 207-243; Graciela Márquez, "Daniel Cosío Villegas, sus años como economista", El Trimestre Económico LXXI, núm. 284 (2004): 877-907. 
JOSÉ RAFAEL GONZÁLEZ DÍAZ

formales de economía, pero en el extranjero. ${ }^{2} \mathrm{Al}$ concluir la Revolución Mexicana se incrementó la demanda de profesionistas especializados en asuntos económicos y financieros para poner en marcha y conducir el desarrollo económico nacional. En este contexto se impulsaron diferentes iniciativas que buscaron sistematizar la enseñanza y la investigación en economía. Los proyectos más relevantes fueron desarrollados por un grupo de jóvenes profesionistas que habían participado como miembros del denominado grupo de los "Siete Sabios" y otros que se agrupaban en la Generación de 1915: "la que se dedicaría, precisamente, a la reconstrucción del país”. ${ }^{3}$

Los primeros economistas mexicanos fueron inspiradores y promotores de múltiples empresas educativas y culturales para reformar la enseñanza de la economía. Se destacan la elaboración de los primeros planes de estudio para una "nueva carrera" de economía, la fundación de centros educativos para la docencia e investigación en economía, el impulso de pioneros y fecundos proyectos editoriales y la formación de la primera generación de expertos mexicanos en economía. Sin embargo, su obra se extiende más allá de los márgenes que hemos indicado y abarcan otras iniciativas.

A su ingenio y visión se deben proyectos como el de la adaptación en México del exitoso curso Contemporary Civilization que se ha impartido sin interrupción en el Columbia College desde el 20 de enero de 1919. ${ }^{4}$ La concepción misma del plan de estudios básico para todos los

${ }^{2}$ En el grupo de agrónomos e ingenieros se encuentran Marte R. Gómez y Alberto J. Pani. En el grupo de contadores, Luis Montes de Oca y Roberto Casas Alatriste. El grupo de los abogados economistas fue más numeroso: Fernando de la Fuente, Manuel Gómez Morín, Gonzalo Robles, Primo Villa Michel, Narciso Bassols, Enrique González Aparicio, Gilberto Loyo, Francisco Zamora, Manuel Mesa Andraca, Martínez Sobral. Entre los autodidactas se encuentran Jesús Silva Herzog, Rafael Nieto y Carlos Díaz Dufoo.

${ }^{3}$ Javier Garciadiego, "Hacia el centenario de la Constitución", discurso de ingreso, 25 de febrero de 2016 (México: El Colegio Nacional, 2016), 35.

${ }^{4}$ Véase: Columbia College, Introduction to contemporary civilization: A syllabus (Nueva York: Columbia University Press, 1919); Timothy Cross, An oasis of order: The core curriculum at Columbia College (Office of the Dean, Columbia College), 175. El curso sigue siendo un aspecto crucial del core curriculum del Columbia College. Véase: Columbia College, acceso el 15 de marzo de 2021, http:/www.college.columbia.edu/core/conciv. El programa 2020-2021 se encuentra en http://www.college.columbia.edu/core/sites/core/files/pages/Contemporary \%20Civilization\%20Syllabus\%202020-2021_0.pdf (acceso el 15 de marzo de 2021). 
estudiantes fue una aportación esencial de la Universidad de Columbia. Este programa se ha considerado "el curso más prestigioso de los planes de estudio de toda la historia estadounidense" ${ }^{5}$ y se convirtió con el tiempo en el marco de referencia de muchos modelos educativos similares en Estados Unidos. El curso se instituyó dos meses después de concluida la Primera Guerra Mundial para enriquecer los requisitos de filosofía e historia de los estudiantes de primer año. La idea era mejorar la comprensión inteligente de los problemas del mundo y sus soluciones, ${ }^{6}$ para alcanzar el verdadero entendimiento integral de sí mismo y de la sociedad. En el mismo sentido lo había formulado uno de los baluartes de la Universidad de Columbia, el profesor John Erskine (1879-1951): "La obligación moral de ser inteligente".?

La idea de adaptar este tipo de cursos en la formación de los economistas fue de Daniel Cosío Villegas (1898-1976); sin embargo, la dirección del proyecto quedó en manos de su discípula, la maestra Consuelo Meyer L'Epée (1918-2010), quien coordinó el esfuerzo inicial de adaptación realizado por un grupo de jóvenes humanistas. ${ }^{8}$

El punto de partida de esta historia fue 1958, cuando la Facultad de Economía de la Universidad de Nuevo León decidió impartir el curso Evolución de la Civilización Contemporánea, que fue la adaptación del curso Civilización Occidental Contemporánea, que se ofrecía en el Columbia College. De este modo los describe Consuelo Meyer, en la presentación que hace del proyecto en el primer tomo de las antologías que prepararon para el curso: "Esta Facultad [de Economía de

${ }^{5} \mathrm{~W}$. B. Carnochan, The battleground of the curriculum (Stanford: Stanford University Press, 1993), 71.

${ }^{6}$ John J. Coss, "Progress of the new freshman course", Columbia University Quarterly (octubre de 1919): 332-334; "The new freshman course", Columbia University Quarterly (julio de 1919): 247-250.

${ }^{7}$ John Erskine, The moral obligation to be intelligent and other essays (Nueva York: Duffield and Co, 1915), 3-34.

${ }^{8}$ Daniel Cosío Villegas, Memorias (México: Joaquín Mortiz, 1976), 306; Consuelo Meyer, "Prólogo" a Evolución de la Civilización Contemporánea. Capítulo I. La herencia clásica: Grecia (México: Comité Editorial del Curso de Civilización Contemporánea, Facultad de Economía, Universidad de Nuevo León, 1963), XXVIII; "Plática con la Srita. Consuelo Meyer", Contrapunto núm. 2 (2007): 23-24; Enrique Krauze, Daniel Cosío Villegas. Una biografia intelectual (México: Tusquets, 2001), 248. 
Nuevo León] tiene una deuda de gratitud con el Lic. Daniel Cosío Villegas, inspirador de la idea de realizar esta obra y colaborador de la misma en muy variadas formas".

Este curso se convirtió en un elemento fundamental del nuevo plan de estudios de la Facultad de Economía (1958). La reforma se ajustaba a las reflexiones que el mismo Cosío Villegas había puesto por escrito en un artículo de carácter académico de 1948, que se titulaba "Errores y soluciones en la enseñanza económica". ${ }^{10}$ Según Cosío Villegas, la enseñanza económica tenía varios retos: el primero consistía en que los alumnos no eran estudiantes de tiempo completo. Otra circunstancia que limitaba era que la enseñanza universitaria en Latinoamérica seguía centrada en el profesor conferenciante, lo que impedía al estudiante ser lo que debe de ser: el verdadero protagonista de su aprendizaje. Dice Cosío Villegas de manera categórica:

En cualquier universidad que se estime existe una regla de cálculo sabida y practicada por todo el mundo, a saber: el estudiante debe leer dos horas por cada hora de clase-conferencia que reciba. Esto sin contar con otra regla no menos importante: la clase-conferencia es constante en los cursos elementales, pero a medida que estos se transforman en superiores, las conferencias disminuyen en número y en importancia, hasta llegar a desaparecer por completo en el curso de seminario o de laboratorio. ${ }^{11}$

Sin embargo, el problema principal para Cosío Villegas estaba precisamente en la posibilidad de integrar la formación especializada en economía con las otras ciencias sociales y humanas. A su juicio, ahí radicaba una de las dificultades de la enseñanza de la economía en nuestros países. Al carecer de un criterio en torno al cual se pudiera organizar la formación del estudiante, la enseñanza quedaba en un estado de perenne desorientación.

La expresión más extrema de esa confusión es la multiplicación de cursos introductorios innecesarios, sin coherencia ni discernimiento.

${ }^{9}$ Meyer, "Prólogo", XVIII.

${ }^{10}$ Boletín del Banco Central de Venezuela VIII, núm. 35-36 (1948): 10-15.

${ }^{11}$ Ibid., 10. 
En este contexto, la integración de los distintos saberes que convergen en la formación del economista era para Cosío Villegas una cuestión urgente, sobre todo, después de las catástrofes que las guerras habían provocado en el mundo. Por consiguiente, no dudó en reconocer los beneficios que ofrecían proyectos como el curso de Civilización Contemporánea: "Se vuelve a clamar por que el estudiante de economía no pierda de vista que vive en un mundo real, tangible y complejo [...] Lo primero ha dado lugar a que se proponga como solución clara y estable el famoso curso de 'civilización contemporánea' de la Universidad de Columbia". ${ }^{12}$

En el mismo texto ${ }^{13}$ propone un plan de estudio de doctorado en economía en el que se incluyen las asignaturas de Civilización Occidental Contemporánea y Civilización Latinoamericana. Así lo atestigua la misma maestra Consuelo Meyer, muchos años después, en una de sus últimas entrevistas: "Se introdujo [en la Facultad de Economía de Nuevo León, en 1958] por sugestión de Don Daniel Cosío Villegas un curso de Civilización Contemporánea que se daba en la Universidad de Columbia de Estados Unidos". ${ }^{14}$

Cosío Villegas no solo se convirtió en animador del proyecto, sino que se involucró en la creación de vínculos institucionales que garantizaran los recursos financieros necesarios para que este se pudiera ejecutar. Así lo había hecho para obtener recursos adicionales para el Colegio de México. Dice Cosío Villegas en sus Memorias:

No vacilamos en acudir a la Fundación Rockefeller y después a la Ford [...] he sido acusado dos o tres veces de haberme vendido al 'Tío Sam' y vendido también al mismísimo Colegio [...] Desde luego, ni yo ni El Colegio hicimos un misterio de que pedíamos y recibíamos esa ayuda, y porque nunca dudé de que era desinteresada y libre de condiciones y aun de vigilancia administrativa. No solo eso, sino que de mi propia iniciativa puse en más de una ocasión a prueba la sinceridad de las intenciones de los funcionarios de esas Fundaciones. ${ }^{15}$

${ }^{12}$ Ibid., 11.

${ }^{13} \mathrm{Ibid}$., 10-15.

14 "Plática con la Srita. Consuelo Meyer", 23-24.

${ }^{15}$ Cosío Villegas, Memorias, 189. 
La Universidad de Nuevo León también recibió este tipo de apoyos. Según el Informe de 1959 de la Fundación Rockefeller, la Facultad de Economía de Nuevo León estaba inaugurando un curso que tenía como objetivo proporcionar a los estudiantes de economía una base sólida de los aspectos sociales, económicos y políticos de la aplicación racional de los conceptos y métodos económicos a las cuestiones de política social. Dice el informe: "El curso de civilización contemporánea es similar a grandes rasgos a los cursos de civilización occidental que se ofrecían en Estados Unidos, pero dando mayor énfasis a las culturas de América Latina y no occidentales". ${ }^{16}$

Consuelo Meyer lo expresa de otra manera: “[La Facultad] deja constancia de su profundo reconocimiento a la Fundación Rockefeller y al Banco de México, S.A., que prestaron su ayuda financiera para la realización de este trabajo". ${ }^{17}$

¿Por qué fueron justo los economistas los que promovieron e introdujeron estos cursos? ¿Qué les ofrecía dentro de la reforma de la enseñanza económica? ¿Cuál era la finalidad del curso de Civilización Contemporánea dentro de estos planes de estudio? ¿En qué medida su inclusión supone una adaptación original? Estas preguntas requieren un análisis de las relaciones entre la ciencia económica y las humanidades a lo largo de la historia de México. Este será el objeto principal de la primera parte de este trabajo. Las preguntas sobre esta primera etapa de incorporación y adaptación del curso de Civilización Contemporánea en la Facultad de Economía de Nuevo León (1958) serán el tema principal de la segunda sección. Lo que parece indiscutible es que este curso se convirtió en la columna vertebral de la reforma de la enseñanza económica en la Universidad de Nuevo León y un motivo de orgullo para la maestra Consuelo Meyer, que lo difundió en distintas universidades de México y América Latina. Así lo expresó: "Yo pensé que ese curso [Evolución de la Civilización Contemporánea] tenía un gran porvenir, lo mandé a diversas universidades de América Latina con el fin de que,

${ }^{16}$ The Rockefeller Foundation, Annual Report 1959 (Nueva York: 1959), 177. La Fundación Rockefeller destinó 35500 dólares para financiar el nuevo curso en la Facultad de Economía y otros 28000 para el Centro de Investigación Social y Económica.

${ }^{17}$ Meyer, "Prólogo", XXIX. 
si se interesaban, lo adoptaran y aprovecharan [...] Puse especial hincapié en matemáticas y estadística, y este curso que era hijo de mi alma de Civilización Contemporánea". ${ }^{18}$

\section{La economía y las humanidades}

El nacimiento de la carrera de economía respondió a la creciente demanda de profesionales especializados en temas económicos y financieros que consolidaran un proyecto de carácter nacional sobre las circunstancias que había dejado el movimiento revolucionario de 1910. Después del conflicto armado la situación del país era complicada y la solución de muchos problemas dependía de la aplicación de los más recientes conocimientos y enfoques profesionales. ${ }^{19}$

Según Cosío Villegas las primeras iniciativas que buscaron instaurar formalmente la enseñanza económica se originaron con el regreso al país de un puñado de intelectuales mexicanos que habían realizado estudios de economía en el extranjero. Se trataba de Miguel Palacios Macedo (1898-1990), ${ }^{20}$ Eduardo Villaseñor (1886-1978), Antonio Espinosa de los Monteros (1903-1959) ${ }^{21}$ y el mismo Daniel Cosío Villegas. Esa primera propuesta se presentó en 1922, durante la gestión de Manuel Gómez Morín (1897-1972) como director de la Escuela Nacional de Jurisprudencia. $^{22}$

En este proceso de creación de instituciones dedicadas a la enseñanza y la investigación en economía fue decisiva la participación de un grupo de jóvenes profesionistas que habían participado como miembros activos del grupo de los Siete Sabios y otros que se agrupan en la denominada Generación de 1915. ${ }^{23}$ El nombre de la Generación de 1915

18 "Plática con la Srita. Consuelo Meyer", 23-24.

${ }^{19}$ Rodríguez Garza y Ávila Sandoval, “La enseñanza y la difusión de la economía”, 207-208.

${ }^{20}$ Miguel Palacios Macedo realizó estudios de economía y filosofía en la Escuela de Altos Estudios de París.

${ }^{21}$ Elena Soto Vargas, "Antonio Espinosa de los Monteros y el Banco de México: Banco Central vs Banco Comercial”, en Históricas Digital (2019): 197-220.

${ }^{22}$ Cosío Villegas, Memorias, 189.

${ }^{23}$ Luis González, Historia de la Revolución Mexicana (México: El Colegio de México, 1979), 143-184; Enrique Krauze, Caudillos culturales en la Revolución Mexicana (México: Siglo XXI, 
JOSÉ RAFAEL GONZÁLEZ DÍAZ

fue utilizado por uno de sus más destacados integrantes, Manuel Gómez Morín, para expresar la identidad de una época:

la esencia de las generaciones debe buscarse en otra parte, en una íntima vinculación establecida entre varios hombres por la existencia en todos ellos, de un mismo impulso inefable, de una inquietud peculiar, de ciertas maneras profundas de entender y valorizar la vida y de plantear los problemas. Es una especie de unidad biológica superior, trascendental; una "consanguinidad" espiritual que se manifiesta lo mismo en las semejanzas que en las diferencias [...] Una generación es un grupo de hombres que están unidos por esta íntima vinculación quizá imperceptible para ellos; la exigencia interior de hacer algo, y el impulso irreprimible a cumplir una misión que a menudo se desconoce, y la angustia de expresar lo que vagamente siente la intuición, y el imperativo de concretar una afirmación que la inteligencia no llega a formular; pero que todo el ser admite y que tiene un valor categórico en esa región donde lo biológico y lo espiritual se confunden. ${ }^{24}$

1976), 11-15. Los miembros del grupo de los Siete Sabios fueron: Antonio Castro Leal (18961981), abogado y rector de la Universidad Nacional de México en el periodo 1928-1929; durante su gestión se creó la Sección de Economía de la Facultad de Derecho y Ciencias Sociales; Alberto Vázquez del Mercado (1893-1980), abogado y ministro de la Suprema Corte de Justicia de la Nación de 1928 a 1931; Vicente Lombardo Toledano (1894-1968), abogado y famoso sindicalista de tendencia marxista que se desempeñó brevemente como gobernador de Puebla entre 1923-1924 y como secretario general de la Confederación de Trabajadores de México (CTM), entre 1936-1941; Teófilo Olea y Leyva (1895-1956), notable abogado, ministro de la Suprema Corte de Justicia entre 1941-1956 y fundador de la Barra Mexicana, Colegio de Abogados; Alfonso Caso (1896-1970), arqueólogo, rector de la Universidad Nacional Autónoma de México (1944-1945), fundador de la Academia Mexicana de Historia y del Instituto Nacional de Antropología e Historia; Manuel Gómez Morín (1897-1972), abogado e ilustre rector de la Universidad Nacional de México (1933-1934), defensor de la autonomía universitaria, miembro del Consejo de Administración del Banco de México, fundador de la Escuela Bancaria y Comercial y fundador del Partido Acción Nacional; Jesús Moreno Baca (1893-1926), abogado y gestor cultural. En la Generación de 1915, además de los anteriores, se incluyen, entre otros, a Miguel Palacios Macedo, Narciso Bassols (1897-1949) y Daniel Cosío Villegas (1898-1976), que también estudiaban en la Escuela Nacional de Jurisprudencia, aunque se matricularon unos años después.

${ }^{24}$ Manuel Gómez Morín, 1915 y otros ensayos (México: Jus, 1973), 26-27. Véase: José Manuel Orozco Garibay, "Manuel Gómez Morín y la educación: su visión de la Universidad", Estudios XI, núm. 105 (2013): 75-106. 
Estos jóvenes compartieron con la generación que les precedió, la del Ateneo de la Juventud, la crítica al positivismo porfiriano y una penetrante inquietud por las humanidades. Sin embargo, su trayecto formativo y sus objetivos tomaron otro cause cuando experimentaron en carne propia el caos y la desorientación que azotó al país durante los años en que transcurría el conflicto revolucionario. ${ }^{25}$

Abandonaron la vocación, exclusivamente literaria, que distinguió a sus predecesores, para embarcarse en la construcción de un programa de acción económica, social, cultural y política para la Revolución. En la variedad de iniciativas que secundaron se esforzaron por unir la rigurosa actividad intelectual con la praxis política. Sin renunciar al "razonamiento especulativo", veían como indispensable la solidez de los métodos de la ciencia y la técnica. ${ }^{26}$

Pretendieron unir el humanismo y la ciencia en concurso indisoluble y subordinarlos a los mandatos de la ética y el compromiso con la nación. Por eso, no resulta extraño que la mayoría de ellos se dedicara, afanosamente, a la tarea de formar instituciones. Las consideraban una pieza clave para el desarrollo de una nación más próspera y equitativa. ${ }^{27}$

La nueva actitud de los jóvenes de la Generación de 1915 estaba relacionada con el rechazo del "cientificismo de antes", del "misticismo vago de los días de lucha y los groseros desbordamientos de un triunfo sin realizaciones". ${ }^{28}$ Heredaron muchos de los valores que distinguieron a sus antecesores, y de manera especial se comprometieron en la defensa de la libertad de cátedra, el estudio riguroso, la pasión por los clásicos griegos y latinos, la filosofía, la historia, las letras y el compromiso con México. ${ }^{29}$

${ }^{25}$ Luz María Uhthoff, "La situación financiera en los años de la revolución, 1910-1920", Iztapalapa núm. 26 (1992): 214-218; véase: Regina Crespo, Itinerarios intelectuales. Vasconcelos y Lobato y sus proyectos para la nación (México: UNAM, 2005), 66.

${ }^{26}$ Humanismo mexicano del siglo XX, comp. por Alberto Saladino García (México: UAEM, 2004), I, 454.

${ }^{27}$ Manuel Gómez Morín, Construcción de instituciones (antología), comp. y estudio introductorio por Carlos Castillo Peraza (México: FCE, 1997), 13.

${ }^{28} \mathrm{Ibid}$., 3.

${ }^{29}$ Luis Mario Schneider, “La generación de 1915: ¿emblema o realidad?”, Boletín II, núm. 2 (1997): 117-127. 
Sin menospreciar la crítica de los miembros del Ateneo a las interpretaciones de la ciencia del positivismo, algunos de los integrantes de la Generación de 1915 cuestionaron el excesivo academicismo de sus precursores y propusieron una técnica humanizada: "la íntima unión de realidad, propósito y procedimiento". ${ }^{30}$ En otras palabras, apreciando el aporte de las humanidades a la formación de los individuos y las sociedades, intentaron dar cause a la técnica guiada por un pensamiento humanista. ${ }^{31}$

El grupo de los Siete Sabios fundó el 5 de septiembre de 1916 la Sociedad de Conferencias y Conciertos con la finalidad de propagar la cultura entre los estudiantes de la Universidad Nacional de México. "Ya para 1919, la generación de 1915 - los llamados 'siete sabios' domina los medios culturales de la capital, es decir, del país. Renovadores del Ateneo, se integran a las propuestas vasconcelistas en un espíritu que parece poner en un solo nivel el quehacer intelectual y la construcción del nuevo país”. 32

Un primer aspecto que llama nuestra atención es que todos ellos eran estudiantes de derecho, pero sus inquietudes se extendieron a otras disciplinas, como la filosofía, los estudios antropológicos, la ética y, sobre todo, la economía. Tres fueron rectores de la Universidad Nacional de México: Antonio Caso, Gómez Morín y Castro Leal, catedráticos distinguidos y funcionarios públicos. ${ }^{33}$

El único aspecto en el que nos detendremos es el modo en que estos miembros de la Generación de 1915 prolongaron la obra de lo que se considera el "mejor Vasconcelos", es decir, el promotor de las grandes empresas culturales del siglo XX. Su actividad incluyó la fundación de instituciones para la enseñanza económica. En 1922, Manuel Gómez Morín analizó la posibilidad de crear una sección de economía

${ }^{30}$ Gómez Morín, "1915”, en 1915 y otros ensayos, 33.

${ }^{31}$ Krauze, Caudillos culturales en la Revolución Mexicana, 109; Elsa Muñiz, Cuerpo, representación y poder: México en los albores de la reconstrucción nacional, 1920-1934 (México: Porrúa, 2002), 161-164.

${ }^{32}$ Silvia Pappe, “Al mar de uno mismo, gotas de poesía”, en José Gorostiza, Poesía y poética, coord. por Edelmira Ramírez (Madrid: ALLCA XX, 1997), 189-190.

${ }^{33}$ Genaro Salinas Quiroga, Los siete sabios de México (México: UnL, 1980), 524. 
en la Facultad de Derecho y Ciencias Sociales, un proyecto que se concretó el 10 de marzo de 1929, el mismo año en el que instauró la Escuela Bancaria del Banco de México. ${ }^{34}$

Por su parte, Daniel Cosío Villegas, que había colaborado con José Vasconcelos en la SEP en la publicación de los Clásicos, ${ }^{35}$ promovió la creación, junto con Manuel Gómez Morín, de la revista el Trimestre Económico, un proyecto que le daba continuidad a la revista Economía (1929-1930), que había dirigido Miguel Palacios Macedo. Años más tarde, creó el Fondo de Cultura Económica, La Casa de España y el Colegio de México. ${ }^{36}$ En este último se estableció más tarde el Centro de Estudios Sociales, dirigido por José Medina Echavarría. ${ }^{37}$

Finalmente, gracias al empuje de Cosío Villegas, se formaron nuevas generaciones de economistas, como Consuelo Meyer y Víctor Urquidi (1919-2004). Cosío Villegas dio vida a la Facultad de Economía de la Universidad de Nuevo León y propuso que se adoptara el curso de Civilización Contemporánea que se impartía en la Universidad de Columbia. ${ }^{38}$

\section{La fundación de la Facultad de Economía de la Universidad de Nuevo León}

La Facultad de Economía de la Universidad Autónoma de Nuevo León se fundó el 27 de septiembre de 1957. El rector en ese momento era Roberto Treviño González (1918-2001), un hombre que se distinguió

${ }^{34}$ Leonardo Lomelí Vanegas, "La Facultad de Derecho y la enseñanza de la economía en México", Revista de la Facultad de Derecho de México LXX, núm. 277 (2020): 533-564.

${ }^{35}$ José Rafael González Díaz, "José Vasconcelos y los 'Grandes libros"”, Estudios XI, núm. 106 (2013): 7-41. Daniel Cosío Villegas colaboró con José Vasconcelos desde el Departamento de Publicaciones que se fundó en la Secretaría de Educación Pública; posteriormente, en el Departamento de Extensión Universitaria y al fin coordinó el semanario La Antorcha cuando Vasconcelos abandonó el país. Véase: Claude Fell, José Vasconcelos. Los años del águila (México: UNAM, 1989), 14.

${ }^{36}$ Víctor Díaz Arciniega, Historia de la Casa. Fondo de Cultura Económica (1934-1994) (México: FCE, 1994), 35-61; véase: Clara Eugenia Lida et al., La Casa de España y el Colegio de México: Memoria, 1938-2000 (México: El Colegio de México, 2000), 41, 134; María Teresa Gómez Mont, Manuel Gómez Morín, 1915-1939 (México: FCE, 2013), 947.

${ }^{37}$ Véase: Víctor Urquidi, “José Medina Echavarría. Un recuerdo”, Estudios sociológicos 4, núm. 10 (1996): 5-10.

${ }^{38}$ Meyer, "Prólogo", IX-XX. 
JOSÉ RAFAEL GONZÁLEZ DÍAZ

por impulsar la renovación de la universidad. A él se debe la fundación de la Escuela de Matemáticas y la Facultad de Economía. ${ }^{39}$ En un principio se designó al contador Ramón Cárdenas Coronado (1909-1993) como responsable principal de la elaboración del primer plan de estudios de la carrera..$^{40} \mathrm{El}$ proyecto se desarrolló en la Facultad de Comercio y Administración fundada tan solo cinco años antes y que en ese momento prosperaba. El crecimiento de la actividad económica en la década de 1940 en Monterrey hacía que se le considerara como la capital industrial de México. ${ }^{41}$

Si esto era cierto, resultaba inexplicable que no se contara con una facultad o escuela de economía, la disciplina a la que, más que ninguna otra, le correspondería auspiciar en su forma más libre y rigurosa el desarrollo de una comunidad. Posteriormente, Ramón Cárdenas Coronado fue nombrado su primer director. ${ }^{42}$ Los líderes universitarios comprendían que la carrera de Economía era fundamental para resolver los problemas sociales del Estado, pero se preguntaban sobre sus posibilidades reales. ¿Cómo y quién podría hacerse cargo de un proyecto tan ambicioso tomando en cuenta los recursos de la universidad? En un primer momento, Cárdenas aceptó la encomienda, consciente de las dificultades de una responsabilidad tan grande, tanto más cuanto que no contaba con los estudios necesarios de economía. Lo primero que hizo fue buscar la orientación de Ángel Santos Cervantes (1905-1975), un banquero de Nuevo León que había sido secretario de Gobierno del Estado y tenía vínculos en los ámbitos económicos y políticos nacionales. ${ }^{43}$ Santos

${ }^{39}$ Apenas unos meses antes, en la sesión ordinaria del H. Consejo Universitario, se había aprobado la iniciativa que creaba la Escuela de Economía.

${ }^{40}$ María Guadalupe Martínez Martínez, "Facultad de Economía: A 45 años de su fundación”, Entorno Económico XL, núm. 239 (2002): 18-26.

${ }^{41}$ Rodrigo Mendirichaga, La cámara industrial de Nuevo León, 1944-1988 (México: Emediciones, 1989), 14.

${ }^{42}$ Ramón Cárdenas Coronado fue el impulsor y fundador de las Facultades de Comercio y Administración y Economía de la Universidad de Nuevo León. Además, fundó el primer centro de investigación en la Facultad de Administración. Cárdenas estudió la carrera comercial en la Academia de Comercio General Zaragoza. Véase: Roberto Chapa, Tiempo y obra. Semblanza de Don Ramón Cárdenas Coronado (México: Universidad Autónoma de Nuevo León, 1985), 11-15.

${ }^{43}$ En 1964, Santos Cervantes fue miembro del Patronato de la Universidad de Nuevo León y posteriormente fue nombrado rector por el gobernador Eduardo Livas. Santos Cervantes nunca aceptó el nombramiento, porque él y Livas se habían enfrentado como precandidatos a la 
lo llevó con Rodrigo Gómez Gómez (1897-1970), otro personaje significativo para la historia de la Facultad de Economía de la Universidad de Nuevo León y para la historia económica de México. ${ }^{44}$ Rodrigo Gómez se desempeñó durante un largo periodo como director general del Banco de México (1952-1970), y desde un principio secundó la idea y ofreció su apoyo moral y económico al proyecto. Hay motivos para que la petición de Cárdenas de crear una carrera de economía hubiera sido recibida con simpatía por Gómez. Además de compartir un origen común, pues los dos eran de Nuevo León, se identificaban porque cada uno había desarrollado una carrera profesional a partir de sus talentos naturales y experiencia, más que de una educación formal. Ambos habían estudiado en una pequeña escuela, la Academia de Comercio General Zaragoza, una de las pocas opciones para prepararse en cuestiones comerciales. Las dificultades y carencias formativas que habían enfrentado les permitió, probablemente, apreciar la relevancia de una sólida preparación académica. ${ }^{45}$ Rodrigo Gómez y Ramón Cárdenas acordaron que el Banco de México enviaría un experto que ayudara con la parte técnica del diseño del plan de estudios. ${ }^{46} \mathrm{~A}$ la postre, la persona que se haría cargo del diseño curricular y su instauración fue Consuelo Meyer,

gubernatura de Nuevo León. La responsabilidad recayó en Eduardo L. Suárez, un egresado de la Facultad de Economía, exalumno que perteneció a la primera generación de graduados de la carrera de Economía de la Universidad de Nuevo León, que había realizado estudios de maestría y doctorado en Yale. A partir de este momento la rectoría de la universidad quedó a cargo de personas con un perfil más técnico que humanístico. Véase: Alfonso Ayala Duarte, "Eduardo L. Suárez, Rector de la UNL (1964-1965)", Enseñanza más Aprendizaje, cuarta época, núm. 5 (2016): 198-200; María Luis Santos Escobedo, Villaldama (México: Universidad Autónoma de Nuevo León, 1998), 36.

${ }^{44}$ Rodrigo Gómez fue un personaje clave en la historia económica de México. Nacido también en Nuevo León, curiosamente estudió en la misma escuela que Cárdenas, la Academia de Comercio General Zaragoza. Sus habilidades como cambista y financiero lo hicieron sobresalir en la banca. Como director general del Banco de México (1952-1970) imprimió su orientación a la banca central mexicana y pudo unir la inquietud por la estabilidad de precios con las preocupaciones por el desarrollo y el bienestar social. Véase: Ma. Eugenia Romero Soleto, "Rodrigo Gómez: Una Banca Central para el desarrollo", Economía UNAM 11, núm. 31 (2014): 53-83.

${ }^{45}$ La Academia de Comercio General Zaragoza fue una institución fundada en 1901 por Anastasio Treviño Martínez (1870-1943) y se convirtió en una de las pocas opciones para la formación de profesionales del ramo mercantil. El conocimiento de las disciplinas comerciales estaba íntimamente vinculado a la experiencia práctica y la teneduría de libros, el oficio que consiste en llevar los libros de contabilidad de una empresa o negocio.

${ }^{46}$ Chapa, Tiempo y obra, 63. 
JOSÉ RAFAEL GONZÁLEZ DÍAZ

pero en ese momento no se podía integrar a la universidad porque gracias a una beca del Banco de México estudiaba en la Universidad de Londres y luego hizo una estancia en Santiago de Chile. ${ }^{47}$ La maestra Meyer se sumó al proyecto un año más tarde, en 1958. Durante ese primer año de la carrera se utilizó un programa de orientación marxista, casi una copia del que estaba vigente en la Escuela Nacional de Economía en la UNAM. ${ }^{48}$ Según la maestra Consuelo Meyer, Rodrigo Gómez pidió consejo a Daniel Cosío Villegas, que en ese entonces fungía como director general del Colegio de México y que como intelectual mexicano había realizado una evaluación de la enseñanza de la economía en México. ${ }^{49}$ Enrique Krauze describe el hecho de este modo: "Ya en 1958, Cosío había promovido la instauración de la carrera de Economía en la Universidad de Nuevo León, con el objeto primordial que de ella surgiesen economistas-intelectuales y no economistas-políticos, de los que él llamaba 'vivillos', 'llegadistas', 'arribistas". 50

La referencia de Krauze está basada en un discurso inaugural del archivo personal de Daniel Cosío Villegas. Con esta información no se puede determinar que la instauración de la carrera de Economía en la Universidad de Nuevo León hubiera sido idea de Cosío Villegas, puesto que el proyecto fue gestado con anterioridad por otros ilustres neoleonenses; sin embargo, no se puede ignorar su influencia en el diseño definitivo de la carrera, en especial por la designación de Consuelo Meyer. En estricto sentido, Meyer era oficialmente una economista delegada por el Banco de México para impulsar la creación de la Facultad de Economía en Nuevo León. Inicialmente llegó con el cargo de consejera académica por cuenta del Banco de México, hasta que

${ }^{47}$ En la entrevista de Ernesto Bolaños Lozano a Consuelo Meyer se pueden conocer las circunstancias en que se dio su colaboración. "La maestra Meyer aprovechó su estancia en Chile para conversar sobre el nuevo proyecto con distinguidos economistas de la Comisión Económica para América Latina y el Caribe, la Universidad de Chile y otras entidades. Con base en estos comentarios y la experiencia propia, formuló un proyecto para la Facultad que ya en México habría de completar don Daniel Cosío Villegas." Ernesto Bolaños Lozano, "La Facultad... hace 50 años: primeros obstáculos", Contrapunto 5 (2005): 7.

${ }^{48}$ Este plan de estudios se estructuró en 1934, en la época de los profundos cambios sociales del sexenio de Lázaro Cárdenas. Véase: Rodríguez Garza y Ávila Sandoval, "La enseñanza y la difusión de la economía", 232-233.

${ }^{49}$ Bolaños Lozano, "La Facultad... hace 50 años", 7.

${ }^{50}$ Krauze, Daniel Cosio Villegas, 248. 
fue designada directora de la institución. Así describe el historiador Roberto Chapa esta etapa en la universidad: "De acuerdo con lo previsto, la Srita. Meyer elabora y propone un cambio radical del plan de estudios que se había adoptado a partir de la fundación de la Facultad, mismo que, después de un amplio estudio y una profunda discusión, es aprobado por el H. Consejo Universitario y puesto en vigor a partir del año lectivo 1958-1959". 51

Consuelo Meyer permaneció cinco años en la universidad, el tiempo que requirió la primera generación para graduarse (1958-1963). Sin lugar a dudas, el proyecto le resultaba estimulante. Animada por Daniel Cosío Villegas y con una experiencia internacional, se impuso a sí misma la misión de consolidar una escuela de economía de calidad internacional. De este modo lo describe ella misma: "Me entusiasmó la idea, no simplemente de impartir cursos de economía en Monterrey, sino de aprovechar la coyuntura de la reciente fundación de la Facultad y contribuir a la formación de la que podría ser una de las mejores escuelas de economía del país y del ámbito latinoamericano". ${ }^{52}$

El proyecto se pudo realizar porque se reunieron todas las condiciones necesarias. Existía la voluntad institucional de impulsar este tipo de formación. ${ }^{53}$ En ese momento se otorgaron, por primera vez en la universidad, becas mensuales en efectivo, para promover el estudio de tiempo completo. Esas becas fueron gestionadas por el contador Ramón Cárdenas Coronado y fueron posibles por el apoyo de la banca, la industria y el comercio del estado. Por otro lado, la reforma de Consuelo Meyer contó con el apoyo técnico e inclusive financiero del Banco de México. Sus protagonistas tenían plena conciencia de que se estaba construyendo uno de los programas más rigurosos de economía del país. Según

${ }^{51}$ Chapa, Tiempo y obra, 64.

${ }^{52}$ Bolaños Lozano, "La facultad... hace 50 años", 9.

${ }^{53} \mathrm{El}$ contexto institucional en el que se instauró el nuevo plan de estudios (1958) era complejo. Realizar un proyecto como el enunciado no era nada fácil. Se tenía que convencer al director, a los estudiantes y a los profesores que ya habían iniciado un año antes con otro plan de estudios. Lo que implicaba un tipo de afectación para los jóvenes que ya se habían matriculado previamente. El rector aconsejó ser muy prudentes, porque era razonable que el nuevo plan de estudios generara descontento. Por eso se permitió a los estudiantes que no se adhirieron al nuevo plan de estudios permanecer en el anterior en el turno nocturno. 
lo advierte María Eugenia Romero Sotelo, la Facultad de Economía de Nuevo León "se convirtió en una fuente importante de formación de funcionarios del Banco Central durante la década de los años sesenta y setenta”. ${ }^{54}$ Además, también resultó decisivo el carácter y la experiencia de sus protagonistas. En ese momento Consuelo Meyer no era una joven inexperta, sino que tenía cuarenta años, había trabajado para el Banco de México y tenía experiencia internacional. Acababa de concluir sus estudios en Inglaterra y aprovechó su estancia en Chile para conversar sobre el nuevo proyecto con distinguidos economistas de la Comisión Económica para América Latina y el Caribe (Cepal), establecida en 1948. Su proyecto era una síntesis del intercambio con diversas entidades y su experiencia personal. Finalmente, la reforma a la Facultad de Economía de la Universidad de Nuevo León aprovechó la visión y experiencia de Daniel Cosío Villegas y la de otros intelectuales vinculados a las ciencias económicas y sociales. Así lo describe Graciela Márquez: "El director del Banco de México, Rodrigo Gómez, no solo aprobó el nombramiento de Consuelo Meyer como directora de la licenciatura en economía, sino que también buscó el apoyo de otros destacados economistas para asesorar dicho proyecto, entre ellos Cosío Villegas y Víctor Urquidi. Ambos intervinieron en el diseño del plan de estudios y entusiasmaron a estudiantes y profesores de otras disciplinas a integrarse a la nueva licenciatura". ${ }^{55}$

Durante muchos años, Cosío Villegas preparó las condiciones para revolucionar la enseñanza de la economía en México. Desde 1942, como director del Departamento de Estudios Económicos del Banco de México, había impulsado un programa de becas en el extranjero, que, a la postre, tuvo un impacto decisivo en la formación de toda una generación de economistas. ${ }^{56}$ Entre los beneficiarios de estas becas se contaron la misma Consuelo Meyer, Mario Ramón Beteta, Jorge Espinosa de los Reyes, Raúl Ortiz Mena. Cosío Villegas también participó en la definición de la Facultad de Economía de Nuevo León, y en ese mismo

${ }^{54}$ Romero Soleto, "Rodrigo Gómez", 79.

${ }^{55}$ Márquez Colín, “Daniel Cosío Villegas”, 900.

${ }^{56}$ Ibid., 899. 
sentido, en la historia de la economía en México. Así lo describe él mismo en este pasaje memorable:

Yo había intervenido activamente en la creación de una escuela de economía dentro de la Universidad de Nuevo León. Redacté los planes de estudio; conseguí del Banco de México que comisionara a Consuelo Meyer para trasladarse a Monterrey y dirigirla; provoqué una primera donación de libros y conseguí los primeros profesores, algunos de ellos extranjeros, pues resultó difícil convencer a los economistas capitalinos cambiar de residencia. Para cuando la escuela quedó bien encaminada, me desentendí de ella. ${ }^{57}$

El influjo de Cosío Villegas en el proyecto de Nuevo León es difícil de determinar, porque indiscutiblemente una empresa de tal magnitud no puede ser obra de una sola persona. Sin embargo, no se puede ocultar la huella que imprimió en casi todos los aspectos. ${ }^{58}$ Uno de los que nos ocupa en este trabajo es el curso de Civilización Contemporánea que le permitía articular la relación de la economía con las otras ciencias sociales y las humanidades. Al respecto dice Consuelo Meyer:

Se introdujo por sugestión de Don Daniel Cosío Villegas un curso de Civilización Contemporánea que se daba en la Universidad de Columbia [...] Entonces recurrí a profesores mexicanos, un pequeño grupo de intelectuales encabezado por el profesor Arturo Cantú [1936-2006], quien había estudiado filosofía aquí en México, para que ellos formaran un comité editorial de los materiales de ese curso que duraría dos años, que iba a sustituir a multitud de cursos introductorios en sociología, economía y otras ciencias; ese curso consistía en estudios y selecciones tomadas de la obra de más de 70 autores de nombre universal, desde la edad clásica griega hasta mediados del siglo xx. ${ }^{59}$

La sugerencia de adaptar este curso de Civilización Contemporánea fue de Cosío Villegas, pero eso no significó una copia o transcripción de lo que se hacía en Columbia, sino que requirió la intervención de un

${ }^{57}$ Cosío Villegas, Memorias, 306.

${ }^{58}$ Rafael Segovia, "Don Daniel en el Colegio", Boletín Editorial núm. 93 (2001): 225-227.

59 "Plática con la Srita. Consuelo Meyer", 23; Meyer, "Prólogo", XVIII. 
grupo de humanistas mexicanos que se encargaron de emprender un verdadero proyecto editorial. Tuvieron que seleccionar textos y preparar materiales de los grandes autores desde la Antigüedad hasta el siglo XX. De ese modo se quería integrar la formación del futuro economista y evitar la dispersión en conocimientos fragmentados y yuxtapuestos. El proyecto era una parte esencial de la formación que otorgaba la misma importancia a la instrucción cuantitativa de las matemáticas y la estadística. Consuelo Meyer estaba convencida de los beneficios que tenía el curso y se encargó de difundirlo:

Yo pensé que ese curso tenía un gran porvenir, lo mandé a diversas universidades de América Latina, con el fin de que si se interesaban, lo adoptaran y aprovecharan el trabajo de este grupo de jóvenes mexicanos, entre los que además de Arturo Cantú, formaban parte el licenciado Lucas de la Garza, que hizo aportaciones muy importantes a ese curso; los hice venir a México a inspeccionar las reservas de la librería más grande de la ciudad, que entonces era Editorial Porrúa, para que seleccionaran toda la bibliografía que les pareciera útil y conveniente para después. ${ }^{60}$

La formación del futuro economista parecía apoyarse en un trípode: el de las disciplinas cuantitativas, entre las que destacaban las matemáticas y la estadística; la preparación especializada y técnica en la ciencia económica; y, finalmente, en la formación humanística e integral. Dice Consuelo Meyer: "puse especial hincapié en matemáticas y estadística, y este curso que era hijo de mi alma de Civilización Contemporánea". ${ }^{61}$

¿Por qué motivo Cosío Villegas sugirió la adaptación de este curso que se impartía en la Universidad de Columbia? Como lo hemos señalado, se percató de que la preparación de los economistas requería un núcleo articulador que le permitiera al estudiante orientarse significativamente en el mundo y responder con sus propios juicios. Esto no se podía lograr colocando cursos introductorios de cada una de las ciencias sociales y humanas que contribuyen a la economía. Se requería algo que integrara al alumno como persona y como futuro profesionista. No

60 "Plática con la Srita. Consuelo Meyer", 23.

${ }^{61}$ Ibid., 24. 
debe extrañarnos que Cosío Villegas tuviera una visión en la que la técnica es inseparable de la historia y las disciplinas humanísticas. Él mismo se declaraba afortunado de haber recibido una formación general y humanística que le servía de base para su preparación técnica profesional. A propósito de su encuentro con Antonio Caso, dijo:

Y estaba en el Centro Antonio Caso [filósofo] en su plenitud: expositor brillantísimo, orador consumado, era, al mismo tiempo, un gran actor, como todo verdadero maestro lo es. Y también como todo maestro excepcional, despertaba en uno el apetito de la lectura y el hábito de reflexionar. Como solía combinar un curso general de historia de las ideas o doctrinas filosóficas con otro que hoy se llamaría monográfico, entonces, no pude ser más afortunado: el curso general me permitió tener un buen panorama de esas doctrinas, es decir, situarme en el universo filosófico, y el segundo, que se repitió al año siguiente, versó sobre ética. ${ }^{62}$

\section{El plan de estudios 1958 de la carrera de economía en la Facultad de Economía de la Universidad de Nuevo León}

El plan de estudios de la Facultad de Economía de Nuevo León (1958) elaborado por Consuelo Meyer y su equipo se publicó el mismo año en el que se puso en práctica. ${ }^{63}$ Se trata de un plan de estudios equilibrado que buscaba la formación rigurosa y técnica de los estudiantes. De

${ }^{62}$ Daniel Cosío Villegas, "Memorias: Cuarto tramo”, en Pensamiento jurídico del Colegio Nacional, coord. por Oscar Ricardo Valero Recio Becerra (México; UNAM / Instituto de Investigaciones Jurídicas / El Colegio Nacional, 2015), 231-232.

${ }^{63}$ Véase: "Facultad de Economía de la Universidad de Nuevo León", El Trimestre Económico 25, núm. 100 (1958): 770. En este documento se realiza una declaración de intenciones de los objetivos y actividades de la Facultad de Economía. Se describen como parte de sus objetivos, entre otros, la formación de economistas versados en los problemas del desarrollo, capaces de ofrecer asesoramiento técnico de la acción pública, de las empresas privadas y de las organizaciones de empresarios y trabajadores, la docencia y la investigación en las esferas académicas, gubernamental y privada y en los distintos organismos internacionales. Además, se determina la creación de un Centro de Investigaciones Económicas que debe ocuparse del desarrollo económico de la región y del país. Finalmente, se le asigna la responsabilidad de difundir conocimientos en las especialidades económicas por medio de publicaciones periódicas. En otras palabras, los objetivos de la Facultad son la docencia, la investigación y la divulgación de la ciencia económica de manera rigurosa y técnica. 
hecho, durante el ciclo 1958-1959 solo se propusieron cinco cursos. ${ }^{64}$ Destacan tres pilares fundamentales. El primero consistía en la búsqueda de una sólida formación cuantitativa, que necesariamente le otorgaba una relevancia al dominio de las matemáticas. De hecho, como dato curioso, se designó como director a Roberto Treviño González, el rector en ese momento de la universidad de Nuevo León. El segundo pilar fue el curso de Civilización Contemporánea, que estaba centrado en el desenvolvimiento de las instituciones e ideas sociales de la cultura occidental. Se trataba de un curso de dos años que imponía a profesores y alumnos tres horas de clase presenciales, y seis horas para la lectura y preparación de textos; es decir, un total de nueve horas a la semana. ${ }^{65}$ Se trataba de un curso de perspectiva humanista e histórica. Por medio de la lectura y el debate se ofrecía la cultura y oportunidades de cultivo de las humanidades. La tercera columna incluía todas las asignaturas estrictamente económicas (para el primer año, solo se impartió una introducción a la economía y otra de contabilidad para economistas). Por último, el proyecto de formación llevaba un curso para el dominio del inglés como lengua extranjera. ¿Qué conclusiones podemos obtener de esta propuesta curricular? Lo primero que debería llamar nuestra atención es la simplicidad de su diseño. Se evitó escrupulosamente la proliferación de cursos y se elaboró una propuesta austera, en la que primara lo esencial. El número de cursos que deberían realizar los estudiantes no era extenso. Se le daba prioridad a la calidad de las asignaturas sobre la cantidad. El espíritu que lo anima es el de la eficacia y la eficiencia. Por eso, se puede decir que es un plan de estudios de corte minimalista. Destinaba los recursos escasos a todo lo que sus proponentes consideraban las cuestiones esenciales de la formación de los economistas. El objetivo primordial era preparar a los estudiantes para recibir el título de licenciado en Economía.

Esta propuesta educativa representa el punto de llegada de un largo proceso de reflexión sobre la enseñanza de la economía al que Cosío

${ }^{64}$ Los cursos que se ofrecieron para los estudiantes del primer año fueron: Introducción a la economía; Matemáticas para economistas; Civilización contemporánea; Contabilidad para economistas e inglés. Ibid., 770.

${ }^{65}$ Ibid., 772. 
Villegas se había dedicado durante los últimos años. Unos diez años antes había redactado su artículo "Errores y soluciones en la enseñanza económica", que se publicó en $1948 .{ }^{66}$ En esa obra lamenta la falta de coherencia e integración de la formación de los economistas, que termina siendo caótica por tantas introducciones que no lograban armonizar la ciencia económica con el resto de las ciencias sociales y humanas. En ese momento ya había contemplado teóricamente que la solución consistía en integrar una formación general en la que pudiera converger, posteriormente, la preparación técnica especializada de la economía. ${ }^{67}$ Cosío Villegas propuso como ejercicio académico un plan (ideal) de doctorado en Economía, que obviamente, en ese momento no se aplicó, pero que fue la base de lo que se hizo posteriormente en Nuevo León.

En el plan de estudios para un doctorado en Economía de 1948 y en el plan de estudios de la Facultad de Economía de Nuevo León de 1958 hay similitudes. La primera es que en los dos se evita sobrecargar de cursos al estudiante. En el doctorado, los cursos no pueden pasar de cuatro por año; en el de licenciatura, de cinco. La segunda semejanza es que en ambos casos se procura una formación de habilidades cuantitativas, matemáticas, análisis estadístico financiero y contabilidad. La tercera semejanza es que toda la formación profesional y técnica en economía se apoya en una formación general. Finalmente, los dos planes proponen como solución al problema de la integración de la economía con el resto de las ciencias sociales y humanas, los cursos de Civilización Occidental Contemporánea. En los dos casos se trataba de cursos que duraban dos años. En el caso del doctorado, la asignatura imponía a profesores y alumnos cuatro clases presenciales, y ocho de lectura y preparación de textos, es decir, doce horas a la semana. ${ }^{68}$ En el doctorado se incorporaba un curso de Civilización Latinoamericana y en el de licenciatura una sección, en el segundo año, con temas análogos. Dice Cosío Villegas en el anteproyecto de 1948: "Al gran problema de centrar la enseñanza de la economía en el conjunto de las ciencias sociales

${ }^{66}$ Cosío Villegas, "Errores y soluciones en la enseñanza económica”, 10-15.

${ }^{67}$ Ibid.

${ }^{68}$ Ibid., 13. 
— problema al cual no se ha ofrecido en ninguna parte solución satisfactoria- se propone la solución de los cursos de Civilización Occidental Contemporánea y Civilización Latinoamericana. El modelo para el primero debe ser el famoso curso de ese nombre que se viene experimentando en la Universidad de Columbia hace ya veintisiete años." ${ }^{69}$

Al respecto, Cosío Villegas cita como fuente principal el artículo de Hacker, "The Contemporary Civilization course at Columbia College"70 y el Manual del libro de fuentes y metodología de estos cursos en la misma universidad. ${ }^{71}$ De este modo describe sus objetivos: "Por eso me conformaré con decir que el primero [Civilización Occidental Contemporánea] tiene por objeto enterar al estudiante de la clase de mundo o de sociedad en que vive, y esto desde el punto de vista no solo económico, sino antropológico, sociológico, político y filosófico". ${ }^{72}$

El segundo curso, de Civilización Latinoamericana, perseguía un objetivo análogo, solo que centrado en que los estudiantes comprendan su realidad inmediata. "El segundo [Civilización Latinoamericana] persigue igual propósito, solo que referido ya a las peculiaridades de la sociedad iberoamericana, con su mixtura de razas y civilizaciones, su geografía y lugar especial en el mundo en general."73

Posteriormente, el mismo Cosío Villegas impulsó la inclusión del curso de Civilización Contemporánea en el plan de estudios de la licenciatura en Economía de 1958 en la Universidad de Nuevo León, lo que logró después de diez años. El comité de Nuevo León describe el origen de los materiales que se estudiarían en el curso de la siguiente manera:

Los materiales del curso están formados por dos antologías, adaptadas en gran parte de materiales publicados por la Universidad de Columbia, que se espera publicar próximamente en español y que en forma provisional han sido reproducidas en mimeógrafo para uso exclusivo de los estu-

${ }^{69}$ Ibid., 14.

${ }^{70}$ América Economic Review XXXV, núm. 2 (1945): 137.

${ }^{71}$ Contemporary Civilization, vol. I: Manual, vol. II: Libro de fuentes (Nueva York: Columbia University Press, 1945).

${ }^{72}$ Cosío Villegas, "Errores y soluciones en la enseñanza económica", 14.

${ }^{73}$ Ibid., 14. 
diantes de la Facultad de Economía: los Ensayos, colección de estudios de carácter expositivo e interpretativo, las Lecturas, colección de textos originales y documentos. Además de estas antologías, los alumnos deberán adquirir La divina comedia de Dante Alighieri y la Breve historia de América de Carlos Pereyra. ${ }^{74}$

En los programas que se incluyen en el plan de estudios de 1958 se declara que el curso de Civilización Contemporánea trata del desenvolvimiento de las instituciones e ideas sociales de la cultura occidental. En el primer año se pretendía estudiar el periodo histórico que se comprende de la Alta Edad Media hasta las primeras décadas del siglo XI. También se incluía una sección para estudiar las guerras de independencia de la América española. ${ }^{75}$ El programa del segundo año del curso no se incluyó, solo se anticipó que abarcaría el estudio de los siglos XIX y XX.

Uno de los aspectos que destaca está en los supuestos didácticos y pedagógicos en torno de los cuales se organizó. A diferencia de los modelos tradicionales, centrados en la cátedra y la conferencia del docente, se demandaba un método completamente diferente en la relación docente-discente. Se declaraba, sin ambigüedad, que la enseñanza del curso exigía un papel activo al estudiante. La lección del profesor se sustituiría por el diálogo en torno a las antologías que se estaban preparando con textos explicativos, originales o documentos. El estudiante tenía la obligación de preparar estas lecturas, previamente señaladas, y presentarse a la clase para discutirlas y expresar sus puntos de vista. Así se describe esta nueva orientación pedagógica: "En la enseñanza del curso [Civilización Contemporánea] se empleará sistemáticamente el método del debate, de manera que será obligatorio para el estudiante presentarse a clase después de haber estudiado cuidadosamente los materiales señalados para discusión y dispuesto a hacer y contestar preguntas, a exponer puntos de vista y a efectuar análisis críticos de los materiales leídos y de las opiniones emitidas en el curso de la discusión”, ${ }^{76}$

74 “Facultad de Economía”, 783.

${ }^{75}$ Ibid., 784-785.

${ }^{76}$ Ibid., 783. 


\section{El curso de Civilización Contemporánea en la Universidad de Nuevo León}

A partir de 1958 la Facultad de Economía de la Universidad de Nuevo León comenzó un intenso proceso de adaptación del curso de Civilización Contemporánea. Según el testimonio de la maestra Consuelo Mayer, la adopción del curso se decidió pocos meses antes de comenzar el ciclo lectivo, 1958-1959. En este breve lapso los miembros de la facultad tuvieron que resolver el problema de traducir más de 2000 páginas de materiales preparados por el Columbia College. Utilizaron la segunda edición de los Chapters in Western civilization y la Introduction to contemporary civilization in the West. ${ }^{77}$

Además de la traducción fue necesario realizar las adaptaciones más esenciales —al menos provisionalmente, en lo que se encontraban otros textos y se reestructuraba de manera original el curso- para los estudiantes latinoamericanos. ${ }^{78}$ Los ajustes fueron continuos, habría que señalar dos que parecen significativos: el primero se relaciona con los periodos históricos que se estudiaban. Inicialmente, en el curso 1958-1959 las antologías comenzaban en la Alta Edad Media y no incluían autores de la Antigüedad. Para 1962 ya se estudiaban autores griegos y romanos. Así lo describe Gustavo Garza:

Cabe destacar que se impartían dos cursos anuales titulados Evolución de la Civilización Contemporánea, I y II. En ellos se estudiaba el devenir del conocimiento desde la edad antigua con la aparición del pensamiento racional iniciado por los griegos (Parménides, Diógenes, Pitágoras, Sócrates, Platón, Aristóteles) y los romanos (Cicerón, Séneca, Catón); la edad media con su mística filosófico-religiosa (Averroes, San Agustín, Santo Tomás de Aquino, Tomás Moro, Calvino, y Erasmo de Róterdam); y la época moderna con el despertar del renacimiento (Descartes, Copérnico,

${ }^{77}$ Contemporary Civilization Staff of Columbia College, Chapters in Western civilization, 2 vols. (Nueva York: Columbia University Press, 1954); Introduction to Contemporary Civilization in the West. A source book (Nueva York: Columbia University Press, 1954), vol. 1, 1250 pp; vol. II, 1281 pp. Ambos textos son la segunda edición.

${ }^{78}$ Meyer, "Prólogo", XVII-XVIII. 
Galileo, Hobbes, Pascal, Spinoza, Locke, Newton, Leibniz, Voltaire, Hume, Rousseau, Montesquieu, Diderot, Kant, Hegel), hasta llegar al positivismo y marxismo del siglo XIX y primera mitad del XX (Schopenhauer, Goethe, Darwin, Malthus, Marx, Engels, Nietzsche y Einstein). ${ }^{79}$

El segundo ajuste tiene que ver con el nombre del curso. En el año 1963, el último en el que Consuelo Meyer fungió como directora de la Facultad de Economía, se lograron publicar los materiales que profesores y alumnos utilizaban para impartir la asignatura. Ya no se habla de Civilización Contemporánea, sino de Evolución de la Civilización Contemporánea. ${ }^{80}$ Además, las lecturas que se incluían en esos volúmenes estaban constituidos por una selecta antología del pensamiento de más de 150 autores antiguos y modernos que de una $\mathrm{u}$ otra forma han influido de manera relevante en la cultura de las sociedades contemporáneas de Occidente. ${ }^{81}$ Entre las lecturas se incluían documentos representativos de algunas etapas en la evolución de estas sociedades.

Los materiales se publicaron con la intención de enriquecer la asignatura con las observaciones de los especialistas y los docentes, y de ese modo, ayudar en "la organización de la llamada educación media o general o el ensanchamiento de la perspectiva cultural y el desarrollo de hábitos de pensar de los estudiantes". ${ }^{82}$ Para la maestra Meyer, el curso era fundamental porque los estudiantes llegaban a las escuelas profesionales "sin un conocimiento adecuado de las ideas e instituciones que, nacidas en el pasado, persisten en la sociedad contemporánea, y sin los instrumentos analíticos que pueden ayudarles a comprenderla mejor". 83

${ }^{79}$ Gustavo Garza, "México: educación pública y formación de un investigador en ciencias sociales”, Estudios demográficos y urbanos 30, núm. 1 (2015). En este texto se reproduce la versión completa del discurso pronunciado por Gustavo Garza en la sesión solemne del H. Consejo Universitario de la Benemérita Universidad Autónoma de Puebla, realizada el 20 de noviembre de 2014, en el cual se le otorgó el grado de doctor honoris causa. En una parte de su disertación valora su estancia en la Universidad de Nuevo León y el sello definitivo que dejó en él la formación recibida cuando tuvo la oportunidad de estudiar la carrera de Economía.

${ }^{80}$ Meyer, "Prólogo", IX.

${ }^{81}$ Ibid., X.

${ }^{82}$ Ibid., IX. Las cursivas son mías.

${ }^{83} \mathrm{Ibid}$. 


\section{Un ambicioso y selectivo proceso editorial}

La implantación del curso de Evolución de la Civilización Contemporánea comenzó con un intenso esfuerzo de selección, traducción y preparación de antologías. Los materiales del curso se distribuían en 26 fascículos cuya extensión en promedio era de unas 150 páginas cada uno. No se trataba solo de una colección de lecturas que se colocaban en una lista cronológica, sino que cada lectura tenía su "propia naturaleza" y quedaba subordina a la estructura general dentro del curso. Cada volumen tenía dos partes: un estudio de carácter expositivo o de interpretación de uno de los temas del curso que tenía el nombre de ensayo, y una selección de textos originales que ilustraban el tema y que eran conocidos como lecturas.$^{84}$ Este es otro de los atributos que hay que destacar. No se trataba de proponer al estudiante lecturas de segunda mano, sino textos originales.

Desde un inicio, los responsables del proyecto captaron que lo más deseable era la preparación de verdaderos ensayos ad-hoc para el curso, pero no contaban con los recursos necesarios y la elaboración de estos textos representaba muchos problemas. Este fue uno de los primeros desafíos. No había posibilidad de tener textos confeccionados a la medida, así que había que buscar afanosamente e ir armando los cursos. ${ }^{85}$

Originalmente, lo que hicieron fue adaptar y traducir una antología preparada por el equipo de Contemporary Civilization del Columbia College ${ }^{86}$ Después, seleccionaron libremente capítulos enteros de la obra de varios autores contemporáneos. Dice Consuelo Meyer: "Solo en raras ocasiones se decidió conservar los ensayos del curso de Columbia College". ${ }^{87}$

Esto quiere decir que el curso de Civilización Contemporánea en México no fue una traducción literal, sino una verdadera adaptación. Casi de inmediato empezó a variar del curso que se enseñaba en Columbia, que tomaba su propio rumbo. Esto no estuvo exento de obstáculos. El comité encargado de adaptar el curso necesitaba

${ }^{84}$ Ibid., XI.

${ }^{85}$ Ibid., X.

${ }^{86}$ Véase nota 79.

${ }^{87}$ Meyer, "Prólogo", XII. 
encontrar textos originales que estuvieran ligados a los temas del curso y que ofrecieran una visión de conjunto en una extensión razonable. Se evitaron los textos fragmentados y se optó por textos "breves" y "coherentes" que pudieran ofrecer al estudiante una visión de conjunto de las problemáticas del curso. ${ }^{88}$ Ese fue uno de los grandes desafíos en esta etapa primigenia de la adaptación y puesta en marcha del curso de Civilización Contemporánea en México. Además, se sumaba a los problemas la singular escasez de materiales en lengua española. Dice Meyer: "La selección de materiales se ha regido por varios criterios. Tratándose de los ensayos, se han preferido los estudios de corta extensión que aúnan a sus cualidades didácticas una visión totalizadora y una interpretación sugestiva del tema en cuestión”, ${ }^{89}$

\section{La influencia del Columbia College}

En la introducción general de los materiales del curso de Evolución de la Civilización Contemporánea de la Facultad de Economía de Nuevo León, los miembros del comité editorial reconocen que el punto de partida de sus antologías era muy semejante al curso de Civilización Occidental Contemporánea que se ofrecía en Columbia desde la década de 1920. El filósofo y educador estadounidense Justus Buchler (1914-1991) elaboró una historia de la evolución del programa del curso de Civilización Contemporánea como parte de la historia de la Universidad de Columbia. ${ }^{90}$ Uno de los cambios más notables ocurrió a partir de 1941, cuando los estudiantes comenzaron a leer selecciones de fuentes primarias, además de artículos especializados y capítulos de libros. Durante el semestre de otoño de ese mismo año, los estudiantes pudieron

${ }^{88}$ Ibid., XI-XII.

${ }^{89} \mathrm{Ibid}$., XII.

${ }^{90}$ Justus Buchler estudió y enseñó en la Universidad de Columbia. En 1971 publicó Theory of human judgment, su primera obra sistemática de filosofía. A lo largo de su vida escribió diversos artículos sobre educación, entre los que destacan: "The liberal arts and general education" (1952), "On the problem of liberal education" (1954), "What is a discussion" (1954) y "Reconstruction in the liberal arts", una historia de la evolución del curso de Civilización Contemporánea como parte de la historia del Columbia College (1954), publicado en Dwight Miner, A history of Columbia College on morningside: The bicentennial history of Columbia University (Nueva York: Columbia University Press, 1954), 48-135. 
comprar sus "libros de consulta" (source books), que contenían las lecturas juntas en un solo paquete. Este cambio hacia la lectura de fuentes primarias representó un fecundo intercambio de ideas dentro del plan de estudios en Columbia. En realidad, el proyecto del curso en la Universidad de Columbia se había originado en 1919. El 20 de enero de ese año, la Facultad de la Universidad de Columbia resolvió remplazar los requisitos de filosofía e historia para los estudiantes de primer año con el curso de Civilización Contemporánea. Se trataba de una clase para grupos pequeños que se reunían una hora cinco veces a la semana. A diferencia de la enseñanza tradicional, en este caso se le daba prioridad a la discusión de los estudiantes por encima del dictado del profesor. Los docentes del curso provenían de los campos de la historia, la filosofía, la antropología e inclusive la sociología. ${ }^{91}$

Sin embargo, no se le había dado coherencia a este proyecto formativo hasta que se logró establecer una fuente única de lecturas para impartir el curso, lo cual solamente se logró con la publicación de Introduction to Contemporary Civilization in the West. A source book en 1946. ${ }^{92}$ Los esfuerzos anteriores para compilar un libro de referencia habían fracasado debido a los desacuerdos entre los profesores sobre lo que se debía incluir. Este libro es el que, inicialmente, tomó la Facultad de Economía de Nuevo León para elaborar su curso de Civilización Contemporánea. De hecho, el primer programa del curso coincide, sin grandes cambios, con el libro de consulta de Columbia de 1946. Sin embargo, durante la adaptación el curso se fue transformando significativamente. Así lo describe el comité editorial de Nuevo León: "solo en raras ocasiones se decidió conservar los ensayos del curso de Columbia College". ${ }^{93}$

Esto confirma que el curso de Civilización Contemporánea en México no fue una traducción literal. Vale la pena hacer una observación, pues frecuentemente se atribuye el surgimiento de estos cursos a la influencia de la Universidad de Chicago sobre algunas universidades

${ }^{91}$ Coss, "The new freshman course", 245-250; "Progress of the new freshman course", Columbia University Quarterly (1919): 332-334.

${ }^{92}$ Véase nota 79.

${ }^{93}$ Meyer, "Prólogo", XII. 
mexicanas. Esta suposición no es exacta, sino que debe tomar en cuenta que el influjo directo provino del Columbia (1919 y 1946). Así como la Universidad de Nuevo León (1958) fue influida por la Universidad de Columbia, lo fue también la Universidad de Chicago. De la Universidad de Columbia provino la inspiración para la obra que realizaron Robert Hutchins (1899-1977) y Mortimer Adler (1902-2001) en Chicago (1930) y los afamados Great Books (1952)..${ }^{94}$ De esta misma Universidad de Columbia surgió el impulso inicial de la Facultad de Economía de Nuevo León (1958). Sin duda, el esfuerzo de reforma en la década de 1930 que realizaron en Chicago Robert Hutchins, Mortimer Adler, Stringfellow Barr (1897-1982) y Scott Milross Buchanan (1895-1968) dio por resultado que se difundiera y obtuviera prestigio internacional la publicación de los 54 volúmenes conocidos como The Great books of the western world (1952). En la Facultad de Economía de Nuevo León el proceso fue paralelo y siguió su propio cause. El modelo de la Universidad de Chicago representado por Robert Hutchins y la concepción de universidad de José Ortega y Gasset (1883-1955), se sintetizarían de modo original, durante la década de 1940, en la Universidad de Puerto Rico bajo el liderazgo del rector Jaime Benítez. ${ }^{95}$

Sobre los contenidos y los objetivos

Los contenidos del curso de Evolución de la Civilización Contemporánea de la Universidad de Nuevo León se agrupaban en torno a las épocas de la historia del mundo occidental y se proponían caracterizar cada época histórica "mediante un cuadro de ideas, instituciones y fuerzas económicas, políticas, religiosas y de otra índole cuya interacción le imparte el carácter de una unidad orgánica”. ${ }^{96}$

Esta interacción de ideas e instituciones referidas a los diferentes contextos no es otra cosa que lo advertido por Wilhelm Dilthey (18331911) sobre que una cosa es la "naturaleza física" o "mundo" y aquello

${ }^{94}$ González Díaz, “José Vasconcelos y los 'Grandes libros”, 10.

${ }^{95}$ Jorge Rodríguez Beruff, "El papel de los estudios generales en los estudios universitarios contemporáneos”, Estudios IX, núm. 97 (2011): 28.

${ }^{96}$ Meyer, "Prólogo", XI. 
que se puede denominar el mundo del espíritu, referido a la historia y la cultura. En palabras de Edmund Husserl (1859-1938): "El mundo circundante histórico [...] no es el mundo objetivo en el sentido nuestro, sino la 'representación del mundo' esto es, su concepción subjetiva del mundo, con todas las realidades para ellos vigentes". ${ }^{97}$

El contenido de este curso estaba centrado en la Weltanschauung (cosmovisión) que distinguió cada etapa de la historia del mundo occidental. El curso de Evolución de la Civilización Contemporánea comenzaba formalmente en la Edad Media y abordaba de manera sucesiva el Renacimiento, la Reforma, el nacimiento del Estado moderno, la Ilustración, el desarrollo de los Estados nacionales y la Edad Contemporánea. ${ }^{98}$ Estas unidades estaban precedidas del estudio, en dos fascículos, de lo más representativo del pensamiento grecorromano como raíces ideológicas de la civilización occidental. La relevancia que adquieren los pensadores de la Antigüedad se debía a que el mismo curso de la Universidad de Columbia fue ampliando la selección de autores antiguos en las ediciones sucesivas de su Introduction to Contemporary Civilization in the West $(1946,1954,1960)$. Es bastante razonable que esta tercera edición haya influido de algún modo en las adecuaciones sucesivas que se hicieron en Nuevo León.

En la selección de las lecturas, el comité de Nuevo León puso el mayor de los empeños en elegir pasajes breves pero que permitieran “conocer el pensamiento, y aun el estilo de los autores representativos de una escuela o de una tendencia dentro de las corrientes del pensamiento económico, político, social o religioso que interesa destacar en cada caso". ${ }^{99}$ Los textos se correspondían con las autores más originales y destacados de la cultura occidental, pero la selección no atendía solo a un contenido fijo e inmóvil de verdades para ser asimiladas por el estudiante, sino que la misma selección se había realizado "atendiendo sobre todo al carácter controvertible de las ideas o teorías expuestas

${ }^{97}$ Edmund Husserl, Invitación a la fenomenología, trad. por Antonio Zirión, Peter Beadet y Elsa Tabernic (Barcelona: Paidós, 1992), 76.

98 "Facultad de Economía", 784-785.

${ }^{99}$ Meyer, "Prólogo", XII. 
en un pasaje determinado, en consonancia con el método de que se valen los maestros para explicar el curso". ${ }^{100}$

Los cursos perseguían dos objetivos, el primero era el desenvolvimiento intelectual del estudiante; el segundo, vinculado con el anterior, era poner al alcance del estudiante, mediante lecturas de gran valor intrínseco de los grandes autores de la historia de Occidente, los medios que le permitan comprender la complejidad de la interdependencia de los seres humanos en la sociedad y los fenómenos sociales. ${ }^{101}$

En relación con el desenvolvimiento intelectual del estudiante, se buscaba que el alumno desarrollara juicios independientes y con sentido de responsabilidad. El alumno tenía que fortalecer su capacidad de análisis, síntesis y expresión crítica. Así quedó expresado en el primer plan de estudios (1958): "La actuación académica del alumno será evaluada sobre la base de la atención y diligencia con que prepare sus clases y de su capacidad de análisis, síntesis y expresión, juzgada por el trabajo que realice en clase en los dos trabajos escritos que se exigirán durante el curso y los exámenes". ${ }^{102}$

El método que se utilizaba para impartir la clase buscaba favorecer el estudio independiente de los alumnos y su activa participación. El estudio de los temas para su discusión obligaba a los estudiantes a abandonar el papel pasivo que de manera tradicional se le asigna a los alumnos en las escuelas por la insana preponderancia de las clases magistrales. Dice Consuelo Meyer:

Al estudiante le es preciso asumir una actitud activa: tiene que mantenerse alerta para seguir el giro que toma la discusión para identificar la idea central o el principio organizador de una teoría o para relacionar hechos e ideas aparentemente distantes, para aducir un argumento o descubrir una consecuencia lógica en el razonamiento de un opositor. De ese modo el estudiante desarrolla su capacidad de análisis, de síntesis y de expresión, al igual que su espíritu crítico y su aptitud para formular juicios independientes y con sentido de responsabilidad. ${ }^{103}$

\footnotetext{
${ }^{100}$ Ibid.

${ }^{101}$ Ibid., XIII-XIV.

102 "Facultad de economía”, 783.

${ }^{103}$ Meyer, "Prólogo", XIII-XIV.
} 
¿Qué quiere decir “desenvolvimiento intelectual”? En este curso se buscaba que los estudiantes tuvieran la capacidad para pensar por sí mismos y emitir juicios fundados sobre los problemas que los afectaban. En cambio, la inteligencia en su versión vulgar se asocia con la capacidad de reproducir una lección o contenido, en casos extremos, aceptando con docilidad las opiniones de un experto. En el ámbito escolar se tiene la pésima costumbre de subordinar la subjetividad de los estudiantes a la voluntad férrea del profesor.

El curso de Civilización Contemporánea buscaba romper con esa inercia y esperaba una reducción gradual de la dirección del profesor hasta volverse algo imperceptible. Solo así el "estudiante alcanzará la plenitud de su capacidad para pensar por sí mismo y enfrentarse a los problemas intelectuales de forma independiente, con lo que quedaría resuelto uno de los grandes problemas de su educación". ${ }^{104}$

El curso ofrecía al estudiante una comprensión de la complejidad de las relaciones del hombre con su entorno y de los fenómenos sociales. Esta concepción coincide con la afirmación de Franklin Baumer (1913-1990) de que los cursos de historia de las ideas permiten a los estudiantes orientarse cósmicamente, lo que Ortega y Gasset descri-

54 bía como la relación entre el pensamiento y las circunstancias. Así lo expresa Ortega:

Una idea es siempre reacción de un hombre a una determinada situación de su vida. Es decir, que solo poseemos la realidad de una idea, lo que ella íntegramente es, si se la toma como concreta reacción a una situación concreta. Es, pues, inseparable de esta, tal vez resulte aún más claro decir esto: pensar es dialogar con la circunstancia. Nosotros tenemos siempre, queramos o no, presente y patente nuestra circunstancia; por eso nos entendemos. Más para entender el pensamiento de otro tenemos que hacernos presentes su circunstancia. ${ }^{105}$

${ }^{104} \mathrm{Ibid}$., XIV.

${ }^{105}$ José Ortega y Gasset, Ideas para una historia de la filosofía (Buenos Aires: Sudamericana, 1942), 391-392. 
El acercamiento del estudiante a las grandes obras ${ }^{106}$ se consideraba una excelente oportunidad para comprender las relaciones de complejidad de las acciones humanas en la sociedad, la interdependencia de los acontecimientos y las concepciones históricas y la complejidad de todos los fenómenos sociales. De este modo lo expresa Consuelo Meyer: "El segundo objetivo del curso consiste en poner al alcance del estudiante, a través de lecturas de gran valor intrínseco, los medios que le permitan entender el papel multifacético que desempeña el hombre en la sociedad, la interdependencia, y la gran complejidad de los fenómenos sociales, los orígenes de numerosas instituciones y, en una palabra, los principales ingredientes de la sociedad en que vive y del juego de fuerzas que la mueven". ${ }^{107}$

En cierto sentido, era un curso fundamental que preparaba para los estudios especializados y profesionales. Una introducción general "al estudio de las ciencias sociales y de las humanidades”. ${ }^{108}$

Esta condición especial lo convertía en objeto de posibles interpretaciones equívocas. Los miembros del comité editorial advirtieron las principales confusiones que podía generar y aclararon su naturaleza y singularidad:

Porque este curso no es muchas cosas. No tiene, por ejemplo, la pretensión de ser una antología literaria, aunque contenga algunos de los pasajes más admirables de la literatura universal; ni una historia de la cultura, por más que presente conjuntos de creencias y tablas de valores que en algunas épocas han regido la vida de los hombres de Europa y América; ni una serie de cuadros que den una visión exhaustiva de ciertas épocas de la historia; tampoco pretende ser una historia de las ideas, si bien aparecen muchas que han dado forma a los módulos culturales contemporáneos; ni una exposición del sistema de pensamiento de autores determinados, pues solo se han presentado las ideas de un autor acerca de un problema particular en un momento dado, y no siempre las más importantes o características, sino las que mejor se han presentado al debate; por último,

${ }^{106}$ González Díaz, “José Vasconcelos y los “Grandes libros””, 9-41.

${ }^{107}$ Meyer, "Prólogo", XIV.

${ }^{108}$ Ibid., XV. 
no es en forma alguna - aunque tal aberración haya sido posible en algunas personas que han examinado superficialmente las antologías- un repertorio de textos destinados a inclinar al lector hacia una postura ideológica, pese a que se exige al estudiante tomar partido cualquiera que este sea, ante las ideas y problemas que se consideran. ${ }^{109}$

\section{La importancia de la participación de los alumnos}

En la reflexión de Consuelo Meyer que acompaña la presentación de los materiales o antologías de la Facultad de Economía de Nuevo León, se advierte de manera enfática que los objetivos del curso, sus contenidos y sus materiales nunca estuvieron desvinculados del método del debate que se empleaba en las clases. Este aspecto que parece secundario, el referido al método, exige una breve reflexión. En realidad, el diálogo, como lo demuestra Georges Gusdorf(1912-2000) en su obra ¿Para qué los profesores?, no es un asunto técnico instrumental, sino que es la esencia del acto educativo. ${ }^{110}$ En el acto educativo se encuentran dos existencias. El profesor asiste el nacimiento de una conciencia que se manifiesta y se hace autoconsciente. Hannah Arendt (1906-1975) afirma que la esencia de la educación es la natalidad. Lo que se manifiesta al profesor es el ser mismo del alumno. Dice Arendt: "la esencia de la educación es la natalidad, el hecho de que en el mundo hayan nacido [auténticos] seres humanos". 111

En el ámbito académico las exigencias del diálogo son relevantes. Se convierte en una insustituible oportunidad de aprendizaje cuando se logran coordinar las actividades que realizan en común los profesores y los alumnos. Dice Arturo Cantú: "Puesto que el trabajo que se hace en clase ha de realizarse fundamentalmente con la participación de los estudiantes, estos han de preparar cuidadosamente cada lectura antes de la clase en que será discutida. De otra suerte es imposible que los fines

${ }^{109}$ Ibid., XV-XVI.

${ }^{110}$ Georges Gusdorff, ¿Para qué los profesores? (Madrid: Cuadernos para el Diálogo, 1969), 196.

${ }^{111}$ Hannah Arendt, "La crisis de la educación", en Entre el pasado y el futuro. Ocho ejercicios sobre la reflexión política (Barcelona: Península, 1996), 270-271. 
del curso se cumplan, como también es imposible tratar de aprender sin haber leído previamente y con atención, con solo escuchar la discusión de quienes sí se han preparado". ${ }^{112}$

Requiere el trabajo de alumnos y profesores para preparar meticulosamente las lecturas y la generosa disposición para analizar críticamente lo leído y sostener juicios personales: "En el curso se emplea el método del debate. El estudiante se presenta después de haber estudiado cuidadosamente los materiales señalados para discusión, y dispuesto a hacer el análisis crítico de los mismos y a exponer y sostener sus puntos de vista en una discusión generalizada que encauza el maestro". ${ }^{113}$

El método requiere, según el comité del curso, la lectura o preparación previa de un texto por parte de alumnos y profesores, y un tipo de clase parecido a la experiencia abierta de diálogo espontáneo y libre. Una especie de seminario (seminarium, etimológicamente "semillero"), como centro de formación y cultivo. La expresión alemana Ausbildung se le asocia en cuanto se refiere al acto de cultivarse uno mismo. En la clase los asistentes interactúan para profundizar en un asunto determinado y pueden darse al debate (disputatio) o confrontación de distintas posiciones. En este sentido, el "método de enseñanza" que se utilizaba en Columbia College y que se usó en la Universidad de Nuevo León y en la Universidad de Chicago no es distinto, en su espíritu, al que se ensayó en su origen en otras universidades, como Oxford, Salamanca y París. ${ }^{114}$ Así lo describe Consuelo Meyer: "El estudiante se presenta a clase después de haber estudiado cuidadosamente los materiales señalados para discusión, y dispuesto a hacer el análisis crítico de los mismos y exponer y sostener sus puntos de vista en una discusión generalizada que encauza el maestro". ${ }^{115}$

Uno de los desafíos más grandes de este tipo de clases está en los criterios y el modo en el que se debe evaluar la participación de los estudiantes. El comité editorial de Nuevo León estableció un criterio al

${ }^{112}$ Arturo Cantú et al., Notas introductorias para los estudiantes (México: Facultad de Economía, Universidad de Nuevo León, 1963), XXII.

${ }^{113}$ Meyer, "Prólogo", XII-XIII.

${ }^{114}$ González Díaz, “José Vasconcelos y los “Grandes libros”, 10 y 14.

${ }^{115}$ Meyer, "Prólogo", XIII. 
respecto: "la participación inteligente de los alumnos en la discusión de los temas asignados, cualquiera que sea la postura que adopten, constituye el criterio básico para evaluar su actuación académica". ${ }^{116}$

Esta forma de concebir la educación requiere una selección y preparación esmerada de los profesores. En el caso de la Facultad de Economía de la Universidad de Nuevo León, se dedicaron esfuerzos institucionales para elegir y preparar a los docentes en este método de enseñanza. Dice Consuelo Meyer: "los maestros encargados del curso en la Facultad de Economía se han preparado especialmente para manejar con soltura este fecundo método de enseñanza". ${ }^{117}$

Después de solventar esta fase inicial, la Facultad de Economía de Nuevo León comenzó un intenso proceso de adaptación más profundo y una preparación más cuidadosa del método de enseñanza para los profesores. Incluso se describe la estancia de uno de los profesores del claustro académico de Nuevo León, para prepararse en el método del diálogo o del debate en otras universidades. Dice Consuelo Meyer:

Pero una vez solucionados los problemas iniciales de la implantación del curso, se presentaba la ineludible necesidad de hacer una adaptación más idónea de los materiales de enseñanza y de preparar profesores de la localidad para la explicación del curso. Esta última tarea se inició en 1959, año en que uno de los profesores de la Facultad tuvo oportunidad de estudiar el método de debate en la Facultad de Estudios Generales de la Universidad de Puerto Rico. ${ }^{118}$

\section{La mirada latinoamericana al curso} de Civilización Contemporánea

La implantación del curso de Civilización Contemporánea en la Facultad de Economía de Nuevo León, que comenzó en 1958, no solo tenía el desafío de traducir el curso de Columbia College y adaptarlo, sino que tenía un reto aún mayor, el de responder de manera inédita a la situación

${ }^{116}$ Ibid.

${ }^{117}$ Ibid., XII.

${ }^{118}$ En el caso de Puerto Rico, las fuentes directas estaban en el pensamiento de Ortega y Gasset y en la experiencia de Robert Hutchins en la Universidad de Chicago. Ibid., XVII. 
que vivían los estudiantes latinoamericanos. Por eso, a la breve fase de implantación se hizo necesaria otra de adaptación profunda. El mejoramiento de los textos no pudo ser iniciado sistemáticamente sino hasta 1961, cuando se constituyó un comité editorial. ${ }^{119}$ Años después, Consuelo Meyer recapitularía este periodo:

Entonces recurrí a profesores mexicanos, un pequeño grupo de intelectuales encabezado por el profesor Arturo Cantú (1936-2006) quien había estudiado filosofía aquí en México, para que ellos formaran un comité editorial de los materiales de ese curso que duraría dos años, que iba a sustituir a multitud de cursos introductorios en sociología, economía y otras ciencias; ese curso consistía en estudios y selecciones tomadas de la obra de más de 70 autores de nombre universal desde la edad clásica griega hasta mediados del siglo XX. ${ }^{120}$

Entonces se formó una comisión permanente encargada de introducir nuevas selecciones para que el curso fuera de mayor interés para alumnos latinoamericanos y norteamericanos. Meyer estaba convencida de que el proyecto tenía un gran porvenir, y como ella misma lo señala, lo envió a diversas universidades del continente con el fin de que las instituciones interesadas lo pudieran adoptar y aprovechar el enorme trabajo que realizaban este grupo de jóvenes intelectuales mexicanos. Dice Meyer: "los hice venir a México a inspeccionar las reservas de la librería más grande de la ciudad, que entonces era Editorial Porrúa, para que seleccionaran toda la bibliografía que les pareciera útil y conveniente para después”. ${ }^{121}$

El comité editorial del curso de Civilización Contemporánea trabajó en el mejoramiento general de los materiales del curso, pero puso particular empeño en ofrecer al estudiante latinoamericano un conjunto de textos que respondieran mejor a sus propias inquietudes y realidades. Aparecen la preocupación por la tradición y cultura hispana,

${ }^{119}$ Ibid.

120 "Plática con la Srita. Consuelo Meyer", 23; Meyer, "Prólogo", XVIII.

121 "Plática con la Srita. Consuelo Meyer", 24; Según el mismo testimonio de Consuelo Meyer: "[El comité] quedó integrado por el profesor Arturo Cantú, como presidente, y por los señores Lic. Héctor Carrasco, Lic. Adolfo Mir y profesor Romero Flores. Ha sido este reducido grupo de jóvenes maestros de la facultad el realizador de la mayor parte de la labor de edición contenida en los primeros 13 fascículos". Ibid., 23; véase: Meyer, "Prólogo", XVII. 
los problemas de la historia y la identidad nacional, y la preocupación por el descubrimiento y la valoración de otras culturas. Dice Meyer: "para ello ha sustituido la casi totalidad de los ensayos del curso de Columbia College por otros que juzgó más adecuados, y ha ampliado muchos pasajes o introducido nuevos ensayos y lecturas referentes a España, a los países latinoamericanos y las civilizaciones de Oriente". ${ }^{122}$

Se incluyeron temas, documentos y otros materiales que abordaban los problemas mundiales que en ese momento eran de la mayor actualidad, entre ellos, el mantenimiento de la paz en el mundo y el desarrollo económico a que aspiran las naciones no industrializadas de la Tierra. Aunque en la presentación de los materiales Meyer sostiene que los nuevos temas y materiales no menguaron el equilibro estructural del curso, podemos vislumbrar que fue mucho más complicado. Los materiales se habían multiplicado y se habían hecho diversos. Integrarlos en un solo curso de dos años debió causar muchos desequilibrios. La naturaleza de la adaptación probablemente reclamaba el surgimiento uno o varios cursos adicionales para que el tratamiento fuera más claro para los alumnos y los profesores. A medida que fue madurando el proyecto, se fue extendiendo hasta demandar otra disposición curricular: "entre los cambios requeridos por estas adaptaciones merece mencionarse la preparación de cuatro nuevos fascículos dedicados especialmente al estudio de España en el momento de la expansión europea, a las revoluciones de independencia y la organización política de los nuevos estados de América, a las culturas orientales y, por último, a los problemas de mediados del siglo XX, considerando la situación de Oriente y Occidente”. ${ }^{123}$

\section{Consideraciones finales}

El curso perseguía que los estudiantes adquieran un conocimiento más profundo de los problemas de su tiempo así como del funcionamiento de la sociedad en que vivían. "Un conocimiento más profundo no implica necesariamente un conocimiento más acucioso de datos históricos

122 "Plática con la Srita. Consuelo Meyer", 23; Meyer, "Prólogo", XVIII.

${ }^{123}$ Ibid. 
o más prolijo de cifras, hechos y nombres. Aunque habrá que prestar alguna atención a los hechos concretos”. ${ }^{124}$ En el curso que implantó y transformó la Universidad de Nuevo León se hacía énfasis en la manera de pensar los problemas, de establecer relaciones entre ellos y de hacer juicios responsables sobre los mismos. El profesor animaba a los estudiantes a llegar a una interpretación personal y libre en diálogo con sus compañeros. Esto no significa una licencia para presentar sus posiciones de modo incoherente o volver la espalda a hechos o ideas que pueda cuestionar sus posiciones. Dice Cantú:

El alumno puede y debe formarse un juicio propio acerca de las ideas y los hechos históricos discutidos en clase, pero este ha de ser un juicio intelectualmente responsable, sustentado en argumentos racionales, atento a todos los hechos o ideas de que tenga conocimiento, y coherentes con los demás juicios que sustente. El fomento de tal libertad para que el estudiante forme sus propias opiniones no tiene otro objeto que el de forzarlo a pensar las cosas por sí mismo, obligarlo a la difícil tarea de pensar por su cuenta dejando de lado memorizaciones de textos pensados por otros autores. Solo en la medida en que el estudiante asuma tal posición activa ante los problemas de la sociedad de que forma parte y ante las herramientas intelectuales necesarias para resolverlos, podrá darse debida cuenta de la magnitud de los mismos, de sus complejidades, de sus implicaciones no aparentes y de todo el esfuerzo humano que la historia ha puesto a su disposición para resolverlos, tanto en experiencia cuanto en concepciones intelectuales. ${ }^{125}$

${ }^{124}$ Cantú et al., Notas introductorias para los estudiantes, XXI.

${ }^{125}$ Ibid., XXII. 
Se prohíbe su reproducción total o parcial por cualquier medio, incluido electrónico, sin permiso previo y por escrito de los editores. 